\title{
Tratamento cirúrgico de múltiplos cistos dentígeros na maxila em paciente pediátrico
}

\author{
Surgical treatment of multiple maxillary dentigeral cysts in pediatric patient \\ Tratamiento quirúrgico de múltiples quistes dentigerales maxilares en paciente pediátrico
}

Recebido: 26/09/2021 | Revisado: 02/10/2021 | Aceito: 13/10/2021 | Publicado: 16/10/2021

\author{
Demóstenes Alves Diniz \\ ORCID: https://orcid.org/0000-0001-5823-6364 \\ Universidade de Pernambuco, Brasil \\ E-mail: dinizdemostenes@gmail.com \\ Lucas Viana Angelim \\ ORCID: https://orcid.org/0000-0001-7596-6027 \\ Universidade Federal de Pernambuco, Brasil \\ E-mail: lucas.angelim@hotmail.com \\ Ana Luíza Ingelbert Silva \\ ORCID: https://orcid.org/0000-0002-0240-2500 \\ Universidade Federal de Pernambuco, Brasil \\ E-mail: al.ingelbert@hotmail.br \\ Thawan Lucas Rodrigues Mendonça \\ ORCID: https://orcid.org/0000-0001-9399-4339 \\ Universidade Federal de Pernambuco, Brasil \\ E-mail: thawanlucasr@gmail.com \\ Vitoria Helena Sales do Nascimento \\ ORCID: https://orcid.org/0000-0003-4111-9393 \\ Universidade de Pernambuco, Brasil \\ E-mail: vitoria.sales@upe.br \\ Caio César Gonçalves Silva \\ ORCID: https://orcid.org/0000-0002-7519-7894 \\ Universidade de Pernambuco, Brasil \\ E-mail: caio.cesar@upe.br \\ Kalyne Kelly Negromonte Gonçalves \\ ORCID: https://orcid.org/0000-0002-8600-9158 \\ Universidade de Pernambuco, Brasil \\ E-mail: kalyne.negromonte@upe.br \\ Francisco Alves de Souza Júnior \\ ORCID: https://orcid.org/0000-0002-3110-2953 \\ Universidade de Pernambuco, Brasil \\ E-mail: fasjunior94@gmail.com \\ Maxsuel Bezerra da Silva \\ ORCID: https://orcid.org/0000-0003-4366-9508 \\ Universidade Federal de Pernambuco, Brasil \\ E-mail:maxsuelmd@hotmail.com \\ Jéssica da Cunha Silva \\ ORCID: https://orcid.org/0000-0003-1570-2964 \\ Universidade de Pernambuco, Brasil \\ E-mail:jc.odonto@gmail.com \\ Rômulo Oliveira de Hollanda Valente \\ ORCID: https://orcid.org/0000-0001-8781-651X \\ Hospital Getúlio Vargas, Brasil \\ E-mail: romvalente@yahoo.com.br \\ Suzana Célia de Aguiar Soares Carneiro \\ ORCID: https://orcid.org/0000-0002-5559-3603 \\ Universidade de Pernambuco, Brasil \\ E-mail: suzanacarneiro@hotmail.com
}

\section{Resumo}

O Cisto Dentígero consiste em uma lesão benigna de origem relacionada com o desenvolvimento odontogênico que circunda a coroa de um elemento dentário não irrompido ao nível da junção cemento-esmalte. Sua patogênese ainda é motivo de discussão, mas acredita-se que seu desenvolvimento seja o resultado do acúmulo de líquido entre o epitélio reduzido do órgão do esmalte e a coroa do dente envolvido. Estudos afirmam que o prognóstico é extremamente favorável quando a lesão é removida por completo, o que evita recidiva e exclui a possibilidade de transformação maligna da lesão. O presente trabalho tem como objetivo relatar um caso de cisto dentígero recorrente em maxila. Discutindo o diagnóstico, tratamento cirúrgico e investigação de causas sistêmicas. O relato de caso, assim como é 
evidenciado pelas pesquisas científicas, mostra a possibilidade desta patologia adquirir grandes proporções, causando diversas interferências na estética e função do paciente. Os cistos dentígeros são lesões relativamente comuns durante a rotina clínica do cirurgião dentista, porém, mesmo que raras, podem apresentar recidivas. Sua presença pode causar diversos problemas estéticos e funcionais ao paciente e, por isso, observa-se a importância dos exames radiográficos para a identificação e o diagnóstico dessas patologias para evitar maiores complicações.

Palavras-chave: Cisto dentígero; Cirurgia maxilofacial; Patologia bucal.

\begin{abstract}
The dentigerous cyst is a benign lesion of origin related to odontogenic development that surrounds the crown of an unerupted dental element at the level of the cementoenamel junction. Its pathogenesis is still a matter of debate, but its development is believed to be the result of the accumulation of fluid between the reduced epithelium of the enamel organ and the crown of the involved tooth. Studies claim that the prognosis is extremely favorable when the lesion is completely removed, which prevents recurrence and excludes the possibility of malignant transformation of the lesion. This paper aims to report a case of recurrent dentigerous cyst in the maxilla. Discussing diagnosis, surgical treatment and investigation of systemic causes. The case report, as evidenced by scientific research, shows the possibility of this pathology acquiring large proportions, causing several interferences in the patient's aesthetics and function. Dentigerous cysts are relatively common lesions during the clinical routine of the dental surgeon, however, even if rare, they can recur. Its presence can cause several aesthetic and functional problems for the patient and, therefore, the importance of radiographic exams for the identification and diagnosis of these pathologies is observed, in order to avoid further complications.
\end{abstract}

Keywords: Dentigerous cyst; Maxillofacial surgery; Oral pathology.

\title{
Resumen
}

El quiste dentígero es una lesión benigna de origen relacionada con el desarrollo odontogénico que rodea la corona de un elemento dentario no erupcionado a nivel de la unión amelocementaria. Su patogenia es todavía un tema de debate, pero se cree que su desarrollo es el resultado de la acumulación de líquido entre el epitelio reducido del órgano del esmalte y la corona del diente afectado. Los estudios afirman que el pronóstico es extremadamente favorable cuando la lesión se elimina por completo, lo que evita la recurrencia y excluye la posibilidad de transformación maligna de la lesión. Este artículo tiene como objetivo reportar un caso de quiste dentígero recurrente en el maxilar. Discutir el diagnóstico, el tratamiento quirúrgico y la investigación de las causas sistémicas. El caso clínico, evidenciado por la investigación científica, muestra la posibilidad de que esta patología adquiera grandes proporciones, provocando diversas interferencias en la estética y función del paciente. Los quistes dentígeros son lesiones relativamente comunes durante la rutina clínica del cirujano dental, sin embargo, incluso si son raros, pueden reaparecer. Su presencia puede ocasionar varios problemas estéticos y funcionales para el paciente y, por ello, se observa la importancia de los exámenes radiográficos para la identificación y diagnóstico de estas patologías, con el fin de evitar mayores complicaciones.

Palabras clave: Quiste dentígero; Cirugía maxilofacial; Patología bucal.

\section{Introdução}

O Cisto Dentígero consiste em uma lesão benigna de origem relacionada com o desenvolvimento odontogênico que circunda a coroa de um elemento dentário não irrompido ao nível da junção cemento-esmalte (Filho et al., 2020; Soares et al., 2018). Apresenta-se como o segundo tipo mais frequente entre os cistos odontogênicos, sendo o mais prevalente quando comparado apenas aos outros cistos de desenvolvimento (Alencar, 2018; Nahajowski et al, 2021; Santos, 2018).

Sua patogênese ainda é motivo de discussão, mas acredita-se que seu desenvolvimento seja o resultado do acúmulo de líquido entre o epitélio reduzido do órgão do esmalte e a coroa do dente envolvido. (Lee, 2020) No entanto, outros estudos mostram que o surgimento dessas lesões pode estar relacionado com a proliferação epitelial em torno da cavidade preenchida fisiologicamente por líquido, que cresce continuamente por pressão osmótica. (Santos, 2019; Vasconcelos, 2017) No entanto, estudos mais recentes afirmam que a gênese do cisto dentígero também pode estar relacionada com uma inflamação apical no antecessor primário, cujas citocinas estimulam a degeneração cística do folículo dentário permanente. (Silveira, 2020)

A sua ocorrência pode estar associada a qualquer dente impactado, porém está relacionada a $24 \%$ dos cistos que envolvem os terceiros molares inferiores, seguidos pelos caninos e terceiros molares superiores, pelos segundos pré-molares inferiores, raramente por dentes supranumerários e incisivos centrais. (Gonçalves \& Rezende, 2020; Kumar et al, 2021; 
Martinelly-Kläy, et al, 2019; Souza, 2020).

Indivíduos do sexo masculino de pele clara são mais comumente citados nas pesquisas como os pacientes mais acometidos por essas patologias. Estas possuem uma predileção pela mandíbula e são encontradas majoritariamente em indivíduos entre a primeira e a terceira década de vida. (Deana \& Alves, 2017; Santos \& Carvalho, 2018)

Por apresentar crescimento lento e assintomático, o diagnóstico pode ser realizado tardiamente, possibilitando uma evolução para uma patologia de maiores proporções, causando abaulamento e adelgaçamento da cortical óssea, assimetria facial, deslocamento dentário, obliteração da cavidade nasal, sintomatologia dolorosa, parestesia do nervo alveolar inferior e até mesmo alterações metaplásicas e displásicas. (Gonçalves \& Rezende, 2020; Nahajowski et al, 2021; Santos \& Carvalho, 2018; Silveira et al., 2020; Soares et al., 2018)

Descoberto em exames radiográficos de rotina ou para auxiliar na investigação do atraso na erupção dentária, o cisto dentígero caracteriza-se como uma imagem radiolúcida unilocular com limites bem definidos, margem esclerótica e halo radiopaco associado às coroas de dentes não erupcionados. Nos casos em que houver infecção, a cortical pode estar ausente. (Amorim, et al., 2021; Benossi, et al., 2019; Martinelli-Kläy, et al., 2019; Souza, 2020)

Os aspectos histológicos evidenciam característica variáveis. Caso não esteja inflamado, possui uma cápsula de tecido conjuntivo frouxo e delgado, revestido por células epiteliais não queratinizadas. No entanto, havendo uma inflamação, o tecido conjuntivo torna-se mais denso com cristas epiteliais hiperplásicas. (Amorim, et al., 2021; Santos, 2019)

Cistos dentígeros geralmente são descritos como lesões solitárias e, se observados bilateralmente ou em múltiplos sítios podem estar associados a alterações sistêmicas, como a Síndrome de Maroteaux Lamy e a Displasia Cleidocraniana. (Alencar, 2018; Santos, 2019). Apesar de raro, o revestimento do cisto dentígero pode sofrer uma alteração neoplásica para um ameloblastoma. (Alencar, 2018; Amorim, et al., 2021)

Após definido o diagnóstico e obtido o resultado de Cisto Dentígero, o tratamento baseia-se nas técnicas de descompressão, marsupialização e enucleação. A escolha de uma única técnica ou da associação entre elas irá depender de alguns fatores, como o tamanho do cisto, localização da lesão, idade do paciente, proximidade de estruturas anatômicas, e a importância clínica do elemento dentário afetado. Após o tratamento definitivo, recidivas ocorrem em casos nos quais ocorre fragmentação do revestimento do cisto e em que ele é deixado no local. Estudos afirmam que o prognóstico é extremamente favorável quando a lesão é removida por completo, o que evita recidiva e exclui a possibilidade de transformação maligna da lesão. (Benossi, et al., 2019; Chouchene et al, 2021; Gonçalves \& Rezende, 2020; Santos, 2019; Souza, 2020)

Dessa forma, o presente trabalho tem como objetivo relatar um caso de cisto dentígero recorrente em maxila. Discutindo o diagnóstico, tratamento cirúrgico e investigação de causas sistêmicas.

\section{Metodologia}

O presente estudo corresponde a um relato de caso, utilizando-se de imagens para seu auxílio e ilustração, sendo conduzido da forma mais tradicional de estratégia de pesquisa, apoiado na técnica da revisão de literatura, integrando-se de um método abrangente de coleta e análise de dados, compreendendo uma completa e clara descrição e discussão de fatores que vão desde o diagnóstico até o tratamento da condição (Pereira et al., 2018). Obedecendo as questões éticas que envolvem este tipo de pesquisa, de forma atinente à resolução no 446/2012 aprovada pelo Conselho Nacional de Saúde, que rege as pesquisas e testes em seres humanos. Este estudo foi aprovado pela comissão de ética do Hospital da Restauração de acordo com a declaração de Helsinque, a responsável legal pela paciente assinou o termo de consentimento livre e esclarecido (TCLE) para participação na pesquisa, autorizando a utilização de dados clínicos-laboratoriais de seu caso clínico/cirúrgico e documentação fotográfica. 


\section{Relato de Caso}

Paciente Sexo masculino, leucoderma, 8 anos de idade, compareceu ao serviço de cirurgia e traumatologia buco maxilo facial do Hospital da Restauração, Recife, Pernambuco, Brasil, com história de cisto dentígero em maxila, já tratado cirurgicamente, três anos posteriormente evoluiu com aumento de volume em região maxilar do lado oposto ao operado (direito) com tempo de evolução de 6 meses.

Figura 1 - Primeiro procedimento cirúrgico para enucleação da lesão.

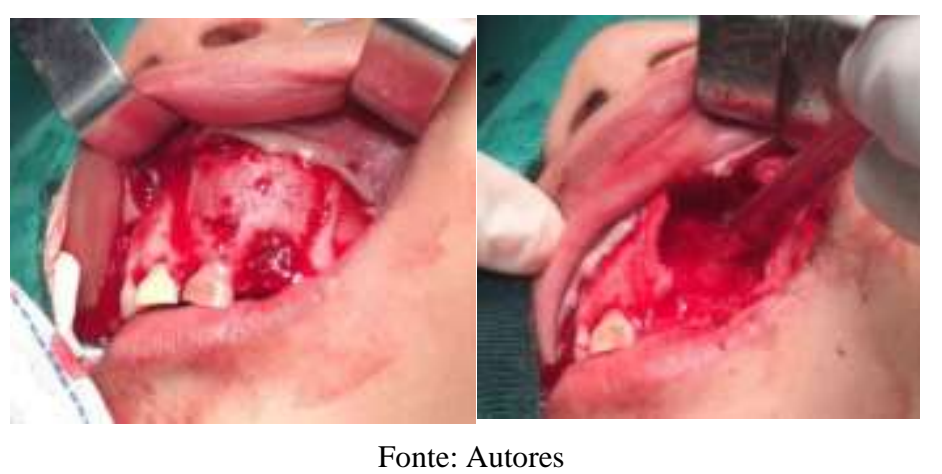

Ao exame físico apresenta tumefação indolor em rebordo maxilar do lado direito, apagamento de sulco geniolabial do mesmo lado, compatível com lesão expansiva, endurecido ao toque, sem ponto de flutuação com características de lesão intraóssea. No rebordo gengival do local da lesão possuía elementos dentários (51, 52 e 53) com mobilidade grau II. Ao realizar punção aspirativa na lesão, observou-se conteúdo citrino, sugestivo de lesão cística.

Figura 2A - Vista frontal do paciente/ Figura 2B- Vista intraoral da lesão evidenciando aumento de volume na região anterior da maxila do lado direito.

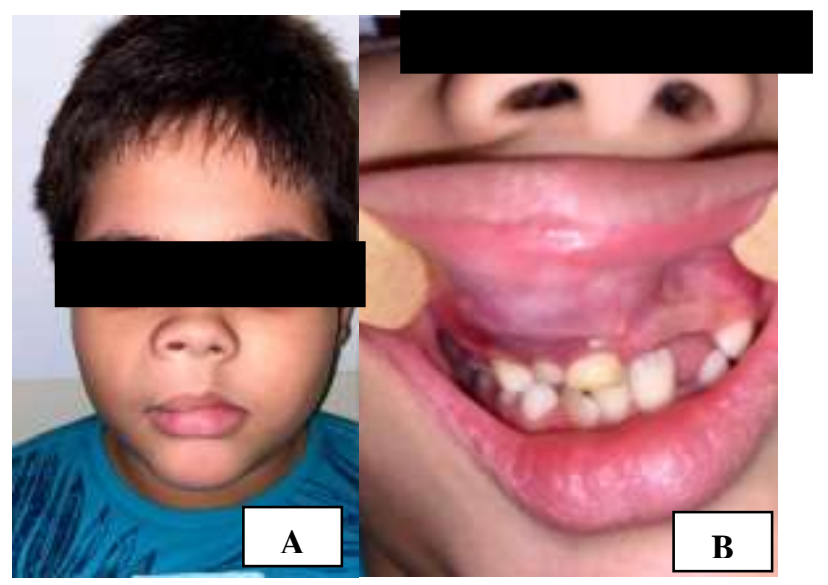

Fonte: Autores.

Foi solicitado tomografia computadorizada da face, do crânio e de tórax, para rastreamento de possível relação sistêmica com a aparição de cistos dentígeros na maxila. Na face, apresentava lesão hipodensa com halo hiperdenso compatível com cisto em maxila, região anterior, associada aos dentes 11, 12 e 13 intra-lesionais. Na tomografia do crânio e do tórax não foram observadas alterações. 
Figura 3A - Reconstrução em 3D/Figura 3B- Tomografia computadorizada de face em corte axial evidenciando lesão hipodensa na região anterior da maxila.

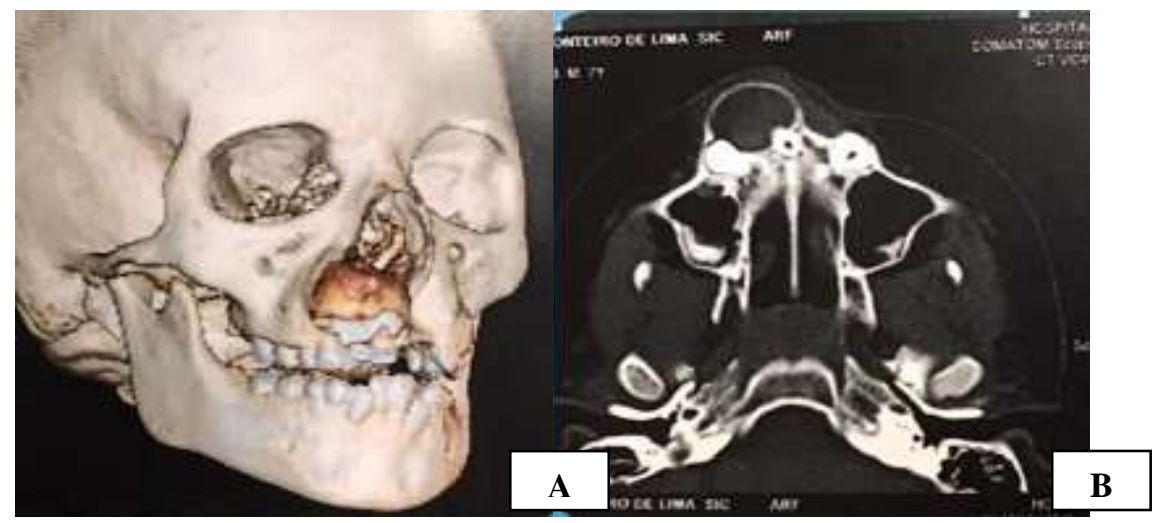

Fonte: Autores.

Assim, o paciente foi submetido à cirurgia sob anestesia geral para tratamento da lesão. Sendo realizado acesso de Newman na região de rebordo alveolar de maxila anterior, enucleação e curetagem da lesão.

Figura 4A - Aspecto clínico da lesão após acesso cirúrgico/Figura 4B- Peça cirúrgica após enucleação/ Figura 4C- Elementos dentários removidos juntamente com a lesão.

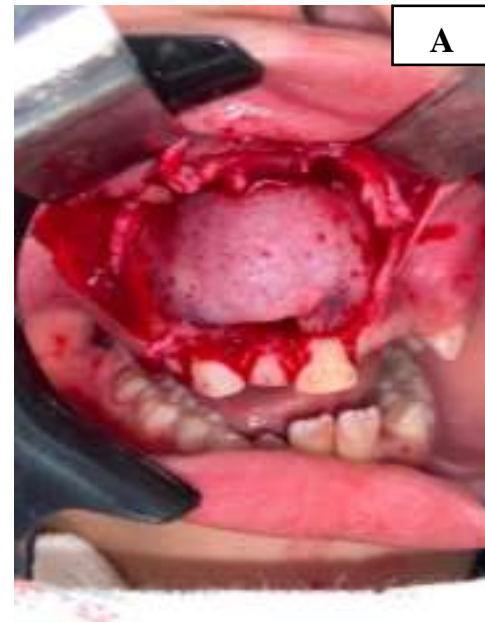

Paciente segue em acompanhamento ambulatorial (Follow Up 6 meses), ausência de sinais de recidiva clínico e radiográficos. Observa-se, ainda, o início da erupção dos elementos dentários mantidos após a enucleação da lesão 
Figura 5A - Reconstrução em 3D/Figura 5B- Tomografia computadorizada de face em corte axial sem a presença da lesão/ Figura 5C- Elementos dentários erupcionando após a remoção da lesão/ Figura 5D- Aspecto extraoral do paciente.

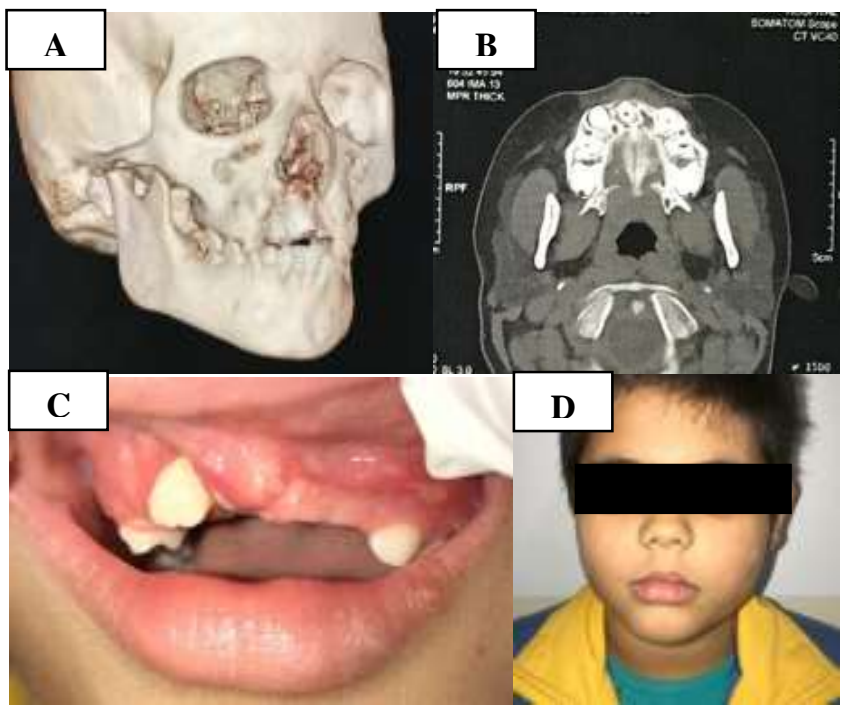

Fonte: Autores.

\section{Resultados e Discussão}

É um consenso o que é afirmado por diversos autores a respeito das características dos cistos dentígeros. Sendo comumente representados como umas lesões benignas relacionadas com o desenvolvimento dos elementos dentários que os envolvem ao nível da junção cemento-esmalte. (Filho, et al., 2020; Santos \& Carvalho, 2018; Soares, et al., 2018). A literatura apresenta informações que são compatíveis com os dados observados no relato de caso exposto, como uma maior prevalência em indivíduos do sexo masculino e de pele clara, e além da idade na qual estas patologias são encontradas. (Deana \& Alves, 2017; Santos \& Carvalho, 2018; Vasconcelos, et al., 2017; Vasiapphan, et al., 2018)

No entanto, algumas informações não são condizentes ao que é expresso na literatura, já que a mandíbula é observada como o osso mais afetado pelo cisto dentígero e, em relação aos dentes, os incisivos centrais superiores não são citados dentre os mais afetados, ficando atrás dos terceiros molares inferiores, caninos e terceiros molares superiores e pelos segundos prémolares inferiores, por exemplo. (Deana \& Alves, 2017; Gonçalves \& Rezende, 2020; Martinelli-Kläy, et al., 2019; Souza, 2020)

O relato de caso, assim como é evidenciado pelas pesquisas científicas, mostra a possibilidade desta patologia adquirir grandes proporções, causando diversas interferências na estética e função do paciente. A literatura ainda afirma que cistos de maiores proporções possuem características únicas, podendo até mesmo sofrer alterações metaplásicas e displásicas e, radiograficamente, podem apresentar uma falsa impressão de lesão multilocular pela presença de trabeculado ósseo no interior da lesão, o que não pôde ser observado no caso descrito neste trabalho, já que mesmo possuindo grande extensão, ainda apresentou aspectos de uma lesão unilocular. (Gonçalves \& Rezende, 2020; Silveira, et al., 2020; Soares, et al., 2018; Souza, 2020)

Ainda observando as características imaginológicas dos cistos dentígeros, eles ainda podem apresentar três diferentes variações: Variante central (quando o cisto envolve totalmente a coroa do dente, que se projeta para o interior da cavidade), Variante circunferencial (quando o cisto envolve a coroa do elemento dentário, estendendo-se ao longo da raiz), e Variante lateral (quando o cisto cresce lateralmente ao longo da superfície radicular, envolvendo parcialmente a coroa do dente) (Alencar, 2018; Amorim, et al., 2021; Benossi, et al., 2019; Soares, et al., 2018; Souza, 2020).

Existe uma alta similaridade entre pequenos cistos dentígeros e folículos pericoronários fisiologicamente mais 
espessos, e por isso faz-se necessário o envio da peça cirúrgica para o exame histopatológico. Alguns estudos apontam que, para que seja considerada uma lesão, o espaço existente deve possuir mais de $3 \mathrm{~mm}$, no entanto já foram encontrados espaços foliculares fisiológicos que possuem essas medidas (Silveira, et al., 2020; Souza, 2020; Lee, 2020)

Assim como pôde ser observado no relato de caso, os cistos dentígeros possuem, mesmo que baixa, a possibilidade de se apresentar como lesões múltiplas e recorrentes. Estudos apontam que pacientes não-sindrômicos esta estatística torna-se ainda mais rara, já que a literatura aponta apenas 18 casos de cistos dentígeros múltiplos em pacientes sem alterações sistêmicas (Devi, et al., 2014).

São duas as principais síndromes associadas com o aparecimento de múltiplos cistos dentígeros. A síndrome de Maroteaux-Lamy advinda da que possui como suas características clínicas a língua alargada, infecções pulmonares, apneia do sono, doenças das válvulas cardíacas, e macrocefalia, além da elevada associação com cistos nos ossos gnáticos. Já a Displasia Cleidocraniana é caracterizada por proeminências dos ossos frontais e parietais, braquicefalia, hipoplasia ou ausência da clavícula, e múltiplas anomalias dentais. (Alencar, 2018; Magalhães, et al., 2019; Medina, et al., 2017; Santos, 2019). Analisando as manifestações clínicas e radiográficas do paciente, o relato de caso deste trabalho não faz referência a um paciente sindrômico.

Além disso, a literatura ainda afirma que por apresentar características similares a outras lesões dos ossos gnáticos, o cisto dentígero possui alguns diagnósticos diferenciais e, por isso, devem ser utilizadas algumas técnicas para a determinação da lesão em questão. A punção aspirativa, por exemplo, pode ser realizada para observar o conteúdo presente no interior da patologia, assim como foi realizado no caso clínico em questão, onde foi obtida a confirmação de uma lesão cística. Uma biópsia incisional prévia pode excluir outras hipóteses diagnósticas como o queratocisto odontogênico e o ameloblastoma, já que estas podem apresentar, em alguns casos, características clínicas e radiográficas semelhantes à cistos dentígeros. (Santos \& Carvalho, 2018; Silveira. et al., 2020; Soares, et al., 2018)

O tratamento escolhido para o caso apresentado neste artigo baseou-se na enucleação e curetagem da lesão para a sua total remoção e para evitar qualquer recidiva. Apesar da literatura afirmar como uma característica é rara, este relato evidência uma recidiva em consequência a realização de uma cirurgia três anos antes. A técnica cirúrgica é de extrema importância e deve ser escolhida com bastante atenção para evitar possíveis danos e resultados indesejados. A idade do paciente, o tamanho da lesão e a sua localização são alguns dos fatores que devem ser levados em consideração para a escolha do tipo de tratamento cirúrgico. (Benossi, et al., 2019; Chouchene et al, 2021; Gonçalves \& Rezende, 2020; Santos, 2019; Souza, 2020)

Lesões menores normalmente são removidas em um único momento cirúrgico, realizando a enucleação cirúrgica. Nos casos de cistos de maiores proporções, a marsupialização pode ser realizada (Técnica de Partsch), assim como a descompressão (Técnica de Thomas) (Alencar, 2018). Estudos apontam que em 85\% dos casos, é realizada a enucleação do cisto e extração do dente não irrompido (Soares, et al., 2018). Porém, observa-se que a realização da enucleação associada à curetagem é uma técnica que pode ser utilizada no tratamento de cistos dentígeros com o intuito de evitar recidivas, como optado no presente caso.

\section{Considerações Finais}

Os cistos dentígeros são lesões relativamente comuns durante a rotina clínica do cirurgião dentista, porém, mesmo que raras, podem apresentar recidivas. Sua presença pode causar diversos problemas estéticos e funcionais ao paciente e, por isso, observa-se a importância dos exames radiográficos para a identificação e o diagnóstico dessas patologias para evitar maiores complicações.

Por fim, o Cirurgião Buco Maxilo Facial possui um importante papel no tratamento destas lesões, já que um bom 
conhecimento das técnicas, assim como o bom emprego das mesmas durante a cirurgia, pode contribuir para um excelente pósoperatório para o paciente, sem recidivas ou danos às estruturas adjacentes.

\section{Agradecimentos}

Os autores agradecem à Coordenação de Aperfeiçoamento de Pessoal de Nível Superior (CAPES), Conselho Nacional de Desenvolvimento Científico e Tecnológico (CNPq) e Hospital da Restauração da cidade de Recife.

\section{Conflito de interesses}

Os autores não têm conflito de interesses a declarar.

\section{Referências}

Alencar, L. M. P. (2018). Cisto dentígero bilateral em mandíbula em paciente não sindrômica: Relato de caso. Universidade Federal do Ceará. 1(1), 1-31

Amorim, K. S., Dantas, A. C. G. C., Nascimento, A. M. S., Rios, M. L. S., Albuquerque Júnior, R. L. C. \& Souza, L. M. A. (2021). Quiste dentígero con transformación ameloblástica. Revista Cubana de Estomatología. 58(1), 234-236.

Benossi, F. I. T., Silva, M. A. E., Salmeron, S., Gottardo, V. D. \& Figueira Júnior, H. C. (2019). Cisto Dentígero: Revisão de literatura e Relato de caso. Revista Uningá. 56(4), 104-112

Chouchene, F., Ameur, W. B., Hamdi, H., Bouenba M., Masmoudi, F., Baaziz, A., Maatouk, F. \& Ghedira, H., (2021) Conservative Approach of a Dentigerous Cyst, Case Reports in Dentistry. 1(1), 6.

Deana, N. F. \& Alves, N. (2017). Cone Beam CT in Diagnosis and Surgical Planning of Dentigerous Cyst. Case Reports in Dentistry. 1(1), 1-7

Devi, P., Bhovi, T. V., Mehrotra, V. \& Agarwal, M. (2014). Multiple Dentigerous Cysts. Journal of Maxillofacial and Oral Surgery. $13(1)$, 63-66.

Filho, M. J. S.F., Andrade, R. L., Prestes, T. P., Cavalcante, F. A., Nascimento, J. R., Aguiar, J. L., Milério, L. R. \& Barros, D. N. R. (2020). A dentigerous cyst associated with a supernumerary fourth molar in the mandibular ramus: a case report. Brazilian Journal of Development. 6(11), 93220-93229

Gonçalves, I. S. M. \& Rezende, J. T. (2020). Cisto dentígero em paciente pediátrico tratado pela técnica da descompressão - Relato de Caso. Universidade de Uberaba. 1(1), 1-16

Kumar M., Malik B., Gothi V. \& Kasrija R. (2021), Marsupialization as Definitive Management of a Large Dentigerous Cyst in an Eleven Year Old Boy: A Case Report, University Journal Of Maxillofacial Surgery Andoralsciences. 1(2), 72-74

Magalhães, A., Meira, J., Cunha, A. M., Moreira, R. J., Leão-Teles, E., Falcão, M., Breda, J. \& Falcão-Reis, F. (2019). Fundoscopic Changes in MaroteauxLamy Syndrome. Case Reports in Ophthalmological Medicine. 1(1), 1-4

Martinelli-Kläy, C. P., Martinelli, C. P., Martinelli, C., Macedo, H. R. \& Lombardi, T. (2019). Unusual imaging features of dentigerous cyst: A case report. Dentistry Journal. 7(76), 1-7

Medina, O., Muñoz, N. \& Moneriz, C. (2017). Displasia cleidocraneal: reporte de un caso. Revista Chilena de Pediatría. 88(4), 514-523

Pereira, A. S. et al. (2018) Metodologia da pesquisa cientifica. UFSM.

Santos, A. V. F. \& Carvalho, R. F. W. (2018) Cisto dentígero: Características clínicas, radiográficas e tratamento - Revisão de Literatura. Universidade Tiradentes. 1(2), 1-15

Santos, L. B. (2019). Tratamento de cisto dentígero: relato de caso. Universidade Tiradentes. 1(1) 1-24

Silveira, A. K. G., Carvalho, L. L. A., Ramos, G. V., Melo, A. S., Grando, W. S. M. S. \& Melo Filho, M. R. (2020). Tubo de polietileno no tratamento conservador de cisto dentígero: Um relato de caso. Brazilian Journal of Health Review. 3(6), 16674-16687

Soares, R. P., Stefanini, A. R., Fabris, A. L. S., Bortoluzzo, P. H. \& Simonato, L. E. (2018). Cisto dentígero: diagnóstico y tratamento. Arch Health Invest. 7(11), 461-464

Souza, B. C. (2020). Cisto dentígero de grande dimensão em maxila: Relato de caso. Journal of Health. 1(1), 193-215

Vasconcelos, M. G., Oliveira, D. H. I. P., Avelar, W. V., Barboza, C. A. G. \& Queiroz, L. M. G. (2017). Localização incomum de cisto dentígero - relato de caso. Odontologia Clínica Científica. 16(4), 315-318

Vasiapphan, H., Christopher, P. J., Kengasubbiah, S., Shenoy, V., Kumar, S. \& Paranthaman, A. (2018). Bilateral Dentigerous Cyst in Impacted Mandibular Third Molars: A Case Report. Cureus. 10(12), 1-7

Udagawa, G., Kataoka, T., Amemiya, K., Kina, H. \& Okamoto, T. (2021). Bilateral kissing molars involving a dentigerous cyst: A case report and literature review. Journal of Oral and Maxillofacial Surgery, Medicine, and Pathology. 1(1), 123-134. 